\title{
ANÁLISE DAS OPORTUNIDADES PARA O DESENVOLVIMENTO MOTOR (AFFORDANCES) EM AMBIENTES DOMÉSTICOS NO CEARÁ - BRASIL
}

\author{
ANALYSIS OF THE OPPORTUNITIES (AFFORDANCES) FOR MOTOR \\ DEVELOPMENT IN THE HOME ENVIRONMENT IN CEARÁ - BRAZIL
}

\author{
Francisco Salviano Sales Nobre ${ }^{1}$ \\ Cícero Luciano Alves Costa ${ }^{1}$ \\ Djevan Lopes de Oliveira ${ }^{1}$ \\ Débora Azevedo Cabral ${ }^{1}$ \\ Glauber Carvalho Nobre ${ }^{1}$ \\ Priscila Caçola ${ }^{2}$
}

NobreFSS, etal. Análise das oportunidades para o desenvolvimento motor (affordances) em ambientes domésticos no Ceará - Brasil. Rev Bras Crescimento Desenvolv Hum. 2009; 19(1):9-18.

\section{Resumo:}

Existe um consenso na literatura científica sobre a forte influência dos estímulos ambientais no desenvolvimento motor das crianças. O presente trabalho tem como objetivo analisar as oportunidades para o desenvolvimento motor em ambientes domésticos de diferentes níveis socioeconômicos no Estado do Ceará, Brasil. Os dados apresentados no estudo foram colhidos a partir das respostas ao questionário Affordances in the Home Enviroment for Motor Development - AHEMD - 18-42 meses quando se entrevistaram 128 tutores responsáveis por crianças com idade entre 18 e 42 meses. Os resultados do estudo são bastante preocupantes, pois mostram uma prevalência da inadequação das estruturas arquitetônicas das residências favorecedoras do desenvolvimento motor e a inexistência de materiais suficientes ao desenvolvimento da motricidade grossa e fina de crianças. Nota-se a necessidade, segundo os dados apresentados, de que seja avaliado o desenvolvimento motor das crianças para verificar se há uma associação entre as affordances e o desenvolvimento motor, e que haja uma aproximação das áreas de engenharia civil, arquitetura e educação física na busca de soluções para este problema. O estudo sugere, ainda, o surgimento de uma nova área de atuação para os profissionais que lidam com o movimento humano no sentido de prestarem consultorias para aquisição de brinquedos que auxiliem no desenvolvimento da motricidade grossa e fina.

Palavras-chave: ambiente; desenvolvimento infantil; classe social.

1 Instituto Federal de Educação, Ciência e Tecnologia do Ceará, IFET CE. Laboratório de Crescimento e Desenvolvimento Motor Humano - LACREDEMH, Juazeiro do Norte, Ceará, Brasil. E-mail: salvianonobre@yahoo.com.br

2 Motor Development Lab, Texas A \& M University, College Station, TX 88743-4243, EUA.

Correspondência para: Francisco Salviano Sales Nobre. Departamento de Educação Física - Instituto Federal de Educação, Ciência e Tecnologia do Ceará - IFT - Brasil - Rua: Plácido Aderaldo Castelo, 1646 - Planalto, Juazeiro do Norte-CE - CEP: 63040-000 - Fone/Fax: (88) 21015300.

E-mail: salvianonobre@yahoo.com.br 


\begin{abstract}
:
Current literature in early childhood emphasizes a strong influence of environmental stimuli on motor skill development. The purpose of the present study was to investigate the opportunities for motor development in different socioeconomic home environments in the state of Ceara, Brazil. The study used the AHEMD (Affordances in the Home Environment for Motor Development) questionnaire with 128 caregivers of children between ages 18 to 42 months. The results appeared to be concerning, showing a prevalence of architectural structures that are unfavorable for motor development, and also for showing an insufficient number of materials that can stimulate gross- and fine-motor development. This study suggests that children's motor development must be assessed in order to detect if there is an association between affordances in the home environment and motor development, and if it is, an approximation of civil engineering, architecture and physical education areas could possibly find solutions for that problem. In addition, it suggests a need of a new career, where professionals who work with human movement can best indicate the acquisition of materials for developing children's gross- and fine-motor coordination.
\end{abstract}

Key words: environment; child development; social class.

\section{INTRODUÇÃO}

Atualmente existe um consenso entre teóricos interacionistas do desenvolvimento humano. Eles advogam que o desenvolvimento se dá a partir de uma constante interação das características do indivíduo com os ambientes em que se encontram inseridos. Os estudiosos do comportamento motor ratificam este posicionamento ao afirmarem que os aspectos individuais (genótipo) e as características socioeconômicas e culturais (fenótipo) contextualizadas pelo ambiente, bem como as tarefas que são realizadas nos diferentes contextos, são determinantes no processo de desenvolvimento motor. ${ }^{1-3}$

Nas perspectivas do Modelo Bioecológico, entende-se que além da relação indissociável entre os atributos da pessoa em desenvolvimento e o contexto em que está inserido, o processo constituído por contínuas estabilizações e mudanças e a ação dos fatores temporais serão determinantes no desenvolvimento deste indivíduo. ${ }^{4-6}$ Considerando que é durante as primeiras idades do indivíduo (com o advento da maturação neurológica) que ocorre uma otimização para a construção de com- portamentos motores necessários à adaptação e exploração do meio, torna-se evidente a influência do contexto imediato vivenciado pela criança para a promoção de um nível de desenvolvimento motor adequado., ${ }^{7,8}$

A esse respeito, o contexto imediato em que a criança se desenvolve é denominado por Bronfenbrenner ${ }^{9}$ como microssistema. Nesse sentido, o microssistema é definido pelo autor como um padrão de atividades, papéis sociais e relações interpessoais vividos pela pessoa em ambientes com características biopsicossociais e simbólicas que convidam, permitem ou inibem o engajamento em atividades progressivamente mais complexas. Sob as perspectivas do modelo bioecológico, fica explícito que as principais relações necessárias para o desenvolvimento motor da criança como cuidados e estímulos são oferecidos pela família no microssistema lar durante a infância. ${ }^{10}$

Entende-se, assim, que tão importante quanto à organização estrutural do ambiente físico é a presença de um agente mediador que facilite o processo de desenvolvimento. Nesses termos, segundo Bronfenbrenner, ${ }^{9}$ agente mediador é todo aquele indivíduo - criança ou adulto, dotado de conhecimento ou experiên- 
cia em uma dada tarefa que ao estabelecer uma relação é capaz de promover o desenvolvimento de outrem. Assim, a qualidade de tais estímulos depende de vários fatores como - o grau de escolaridade dos pais ou responsável (pessoa que passa maior parte do tempo com a criança); a presença de outros adultos além dos pais; a interação com outras crianças e a condição estável de vida da família (a renda mensal em relação ao custo de vida da região onde reside). ${ }^{7}$

Ainda sobre esse assunto, é entendido que fatores como a tipologia dos espaços existentes nas casas, os tipos de solos, a variedade de brinquedos e objetos, a roupa que usa, a presença ou não de irmãos, as práticas dos parentes ou de pessoas que vivem no ambiente da criança, o nível socioeconômico, entre outros, constituem fatores intervenientes nas oportunidades que conferem desafios ao indivíduo em desenvolvimento. ${ }^{7,8,11}$ Nesse sentido, as ações promovidas em resposta aos estímulos podem subsidiar a formação do repertório motor da criança na aquisição de determinada habilidade.

Partindo do conceito de Affordance, utilizado para denominar as oportunidades que conferem desafios ao indivíduo em desenvolvimento, as ações promovidas em resposta aos estímulos podem subsidiar a formação do repertório motor da criança ${ }^{7}$ e, considerando as disparidades socioeconômicas presentes no município de Juazeiro do Norte, Ceará, Brasil, que tão bem exemplifica o problema de má distribuição de renda no País, o presente estudo teve como objetivo analisar as oportunidades para a estimulação motora no ambiente doméstico de crianças entre 18 e 42 meses.

\section{MÉTODO}

Esta pesquisa se caracteriza como sendo um estudo descritivo, transversal, quantita- tivo e de campo. A amostra foi voluntária, por disponibilidade, do tipo não probabilística e intencional, constituída por 128 entrevistados de diferentes níveis socioeconômicos, responsáveis pela tutela de crianças com idade entre 18 e 42 meses. $O$ contato com os entrevistados se deu através de visita a domicílios localizados em três bairros distintos da referida cidade. Utilizou-se como critério de inclusão na pesquisa que nos lares residisse pelo menos uma criança com idade entre 18 e 42 meses e que os responsáveis pela tutela estivessem no domicilio. Após a explicação dos objetivos e consentimento sobre a participação no estudo, os sujeitos eram instruídos sobre o preenchimento do questionário. Nos casos em que estes eram analfabetos ou semi-analfabetos, os pesquisadores faziam a leitura e explicação do instrumento e só então os entrevistados o respondiam.

Este estudo foi desenvolvido em Juazeiro do Norte, município localizado na região Sul do estado do Ceará, Nordeste brasileiro. Os últimos dados, divulgados pelo Instituto de Pesquisa e Estratégia Econômica do Ceará $^{12}$, apresentam a cidade com uma população de 224.141 habitantes; o Produto Interno Bruto (PIB) de R \$ 481,3 milhões e PIB per capita de R\$2.186 por ano. No entanto, o Índice de Desenvolvimento Humano (IDH) do referido município é de 0,397 , o que comprova os problemas sociais existentes. Assim, nos lares investigados, por exemplo, ao se constatar que $79,8 \%$ possuem uma renda mensal inferior a $\mathrm{R} \$ 1.000$, confirma-se um problema típico brasileiro - a má distribuição de renda.

O instrumento utilizado para avaliar o nível de oportunidades para o desenvolvimento motor das crianças foi o questionário Affordances in the Home Enviroment for Motor Development - AHEMD - 18-42 meses. ${ }^{13}$ Trata-se de um questionário com a parte inicial destinada à identificação das características 
da criança e família, e 67 perguntas relacionadas ao ambiente familiar, sendo dividido em cinco subescalas: espaço exterior, espaço interior, variedade de estimulação, material de motricidade fina e material de motricidade grossa.

Após a aplicação do questionário, os dados coletados foram introduzidos e classificados com o auxílio de uma aplicação do programa Microsoft Excel (AHEMD Calculador VPbeta1.5.xls), construído pelos idealizadores do Projeto AHEMD e disponibilizado no endereço eletrônico (http:www.ese.ipvc.pt/ dmh/ AHEMD/ahemd. htm). Para efeito de comparação e a fim de verificar possíveis influências do fator econômico sobre aspectos relacionados ao lar, os lares submetidos ao estudo foram divididos em dois grupos: Grupo A - formado por lares com renda mensal abaixo de R \$1.000; e o Grupo B -constituído por lares cuja renda familiar mensal era superior a R\$1.000.

Foi utilizada estatística descritiva, com medidas de tendência central de distribuição de freqüência; e inferencial, o teste Qui-Quadrado para os dados categóricos. Além do tes- te U de Mann-Whitney para verificar possíveis diferenças no escore total entre grupos. O nível de significância adotado em ambos os testes foi de $5 \%$.

\section{RESULTADOS E DISCUSSÕES}

Considerando que o estatuto socioeconômico, o nível intelectual dos pais e a prematuridade são reconhecidos como fatores intervenientes nas oportunidades facilitadoras para um efetivo desenvolvimento motor ${ }^{10,11,14}$, são apresentados, na tabela 1 , os dados da amostra relacionados ao grau de escolaridade do pai e da mãe, e à renda familiar.

Como pode ser verificado na tabela 1 , o nível econômico é determinante nas melhores condições de escolaridade dos tutores, registrando-se, inclusive, diferenças significativas a favor dos pais e das mães de lares com maior renda. O estudo revela, também, uma tendência evidenciada na literatura $^{15,16}$, apontando para um melhor nível de escolaridade das mulheres, independentemente do nível econômico. Essas informações parecem ser pertinentes, uma vez que as características do agen-

Tabela 1: Distribuição de freqüência da escolaridade dos pais em função da renda mensal familiar.

\begin{tabular}{|c|c|c|c|c|c|c|c|}
\hline & Grupo & $\begin{array}{l}\text { Nunca } \\
\text { estudou }\end{array}$ & $\begin{array}{l}1^{\circ} \text { à } 4^{\circ} \\
\text { série }\end{array}$ & $\begin{array}{l}5^{\circ} \text { à } 8^{\circ} \\
\text { série }\end{array}$ & $\begin{array}{l}\text { Ensino } \\
\text { médio }\end{array}$ & $\begin{array}{l}\text { Ensino } \\
\text { superior }\end{array}$ & \\
\hline \multirow{3}{*}{ Escolaridade do pai } & $\mathrm{A}$ & (02) & (29) & (41) & (30) & (01) & \multirow{3}{*}{$0,001^{*}$} \\
\hline & \multirow{3}{*}{ B } & $1,9 \%$ & $28,2 \%$ & $39,8 \%$ & $29,1 \%$ & $1,0 \%$ & \\
\hline & & - & (03) & (03) & (13) & (04) & \\
\hline \multirow{9}{*}{ Escolaridade da mãe } & & & $11,5 \%$ & $23,1 \%$ & $50,0 \%$ & $15,4 \%$ & \multirow{9}{*}{$0,000^{*}$} \\
\hline & TOTAL & $(02)$ & (32) & (47) & (43) & (05) & \\
\hline & & $1,6 \%$ & $24,8 \%$ & $36,4 \%$ & $33,3 \%$ & $33,3 \%$ & \\
\hline & A & - & (22) & (42) & (35) & (04) & \\
\hline & & & $21,4 \%$ & $40,8 \%$ & $34,0 \%$ & $3,9 \%$ & \\
\hline & B & - & (01) & (02) & (18) & (05) & \\
\hline & \multirow{3}{*}{ TOTAL } & & $3,8 \%$ & $7,7 \%$ & $69,2 \%$ & $19,2 \%$ & \\
\hline & & & (23) & (44) & (53) & (09) & \\
\hline & & & $17,8 \%$ & $34,1 \%$ & $41,1 \%$ & $7,0 \%$ & \\
\hline
\end{tabular}

* Diferenças significativas para $\mathrm{p}<0,05$ (teste Qui-quadrado) 
te mediador constituem um fator determinante na qualidade dos estímulos favorecedores do desenvolvimento. ${ }^{7,9,10}$
Segundo as observações anteriormente citadas, a tabela 2 mostra características de infraestrutura física, ação do agente mediador na estimula-

Tabela 2: Distribuição de freqüência da classificação das sub-escalas do affordances in the home enviroment for motor development (AHEMD) de acordo com a renda mensal familiar e total.

\begin{tabular}{|c|c|c|c|c|c|c|c|}
\hline & GRUPO & MUITO & MUITO & FRACO & BOM & MUITO & $\mathbf{p}$ \\
\hline & & FRACO & FRACO & & & BOM & \\
\hline \multirow[t]{5}{*}{ Espaço Exterior } & A & (55) & (55) & $(40)$ & $(06)$ & $(02)$ & \\
\hline & & $53,4 \%$ & $53,4 \%$ & $38,8 \%$ & $5,8 \%$ & $1,9 \%$ & \\
\hline & B & (11) & (11) & $(07$ & $(07)$ & $(01)$ & $0,013 *$ \\
\hline & & $42,3 \%$ & $42,3 \%$ & )26,9\% & $26,9 \%$ & $3,8 \%$ & \\
\hline & TOTAL & (66)51,2\% & (66) $51,2 \%$ & $(47) 36,4 \%$ & (13)10,1\% & (03)2,4\% & \\
\hline \multirow[t]{5}{*}{ Espaço Interior } & A & (24) & (24) & (11) & (15) & (53) & \\
\hline & & $23,3 \%$ & $23,3 \%$ & $10,7 \%$ & $14,6 \%$ & $51,5 \%$ & \\
\hline & B & $(7,7)$ & $(7,7)$ & $(01)$ & (10) & (13) & $0,022 *$ \\
\hline & & $7,7 \%$ & $7,7 \%$ & $3,8 \%$ & $38,5 \%$ & $50,0 \%$ & \\
\hline & TOTAL & ـ (26) $20,2 \%$ & (26)20,2\% & $(12) 9,3 \%$ & (25)19,4\% & (66)51,2\% & \\
\hline Variedade de & A & (13) & (13) & (19) & (23) & (48) & \\
\hline \multirow[t]{4}{*}{ Estimulação } & & $12,6 \%$ & $12,6 \%$ & $18,4 \%$ & $22,3 \%$ & $46,6 \%$ & \\
\hline & B & (06) & (06) & (06) & $(02)$ & (12) & 0,254 \\
\hline & & $23,1 \%$ & $23,1 \%$ & $23,1 \%$ & $7,7 \%$ & $46,2 \%$ & \\
\hline & TOTAL & 年) 14,7\% & (19)14,7\% & (25)19,4\% & (25)19,4\% & (60)46,5\% & \\
\hline Material de & A & (95) & (95) & $(07)$ & (01) & - & \\
\hline Motricidade & & $92,2 \%$ & $92,2 \%$ & $6,8 \%$ & $3,8 \%$ & - & \\
\hline \multirow[t]{4}{*}{ Fina } & B & (21) & (21) & $(04)$ & (01)1.6\% & & 0,201 \\
\hline & & $80,8 \%$ & $80,8 \%$ & $15,4 \%$ & - & (01) & \\
\hline & TOTAL & (116)89,9\% & (116)89,9\% & (11)8,5\% & - & $1,0 \%$ & \\
\hline & A & (98) & (98) & $(04)$ & - & - & \\
\hline Material de & & $95,1 \%$ & $95,1 \%$ & $3,9 \%$ & $11,5 \%$ & (01) & \\
\hline Motricidade & B & (23) & (23) & (03) & $(07) 5,4 \%$ & - & 0,273 \\
\hline \multirow[t]{2}{*}{ Grossa } & & $88,5 \%$ & $88,5 \%$ & $88,5 \%$ & & - & \\
\hline & TOTAL & (121)93,8\% & (121)93,8\% & (121)93,8\% & & - & \\
\hline
\end{tabular}

*Diferenças significativas para $\mathrm{p}<0,05$ (teste Qui-quadrado) 
ção motora e a disponibilidade de recursos materiais, como estratégia de desenvolvimento da motricidade grossa e fina. Para tanto, os dados obtidos das cinco subescalas foram classificados numa escala do tipo Likert, categorizada da seguinte forma: "muito fraco", "fraco", "bom" e "muito bom", e analisado sob o enfoque da renda familiar.

No que diz respeito às subescalas - espaço exterior e espaço interior, as que analisam o espaço físico interno e externo, aparatos internos e externos, superfícies internas e espaços internos para brincadeiras existentes no $\operatorname{lar}^{17}$, verificou-se que, independentemente do nível econômico, a classificação foi muito fraca e fraca para a variável espaço exterior; o mesmo não ocorrendo no item espaço interior, de que ambos os grupos obtiveram classificação boa e muito boa, havendo no entanto uma melhor classificação para o grupo de melhor poder aquisitivo nas duas variáveis. Os achados evidenciados neste estudo vão ao encontro do que também foi observado no estudo de Müller ${ }^{11}$ em Porto Alegre, na região sul do País, onde se verificou que o espaço exterior de mais da metade das residências analisadas não oferecia oportunidades suficientes para o desenvolvimento motor das crianças.

Esses dados indicam a necessidade de se promover uma aproximação das áreas de engenharia civil, arquitetura e educação física, especificamente na área de desenvolvimento motor. Juntas essas três áreas de estudo oferecerão orientações para a elaboração de estratégias arquitetônicas que melhor estruturem o espaço físico das residências e espaços públicos. Nem mesmo as residências com perfil de nível socioeconômico elevado apresentaram características que favoreçam um desenvolvimento motor das crianças de forma adequada.

Uma vez que parte da estrutura ambiental não favorece o desenvolvimento motor das crianças, fica explícita a importância da intervenção do agente mediador no microssistema lar para suprir essas necessidades. No entanto, contrariando todas as expectativas evidenciadas na literatura ${ }^{10,11,14}$ que apontam para uma influência do estatuto socioeconômico e do nível intelectual dos pais sobre a qualidade dos estímulos nas oportunidades facilitadoras para um efetivo desenvolvimento motor, constatou-se que no item variedade de estimulação, 68,9\% do Grupo A apresentaram uma classificação boa e muito boa, contra 53,9 \% do Grupo B. No total, $65,9 \%$ dos lares obtiveram classificações boa e muito boa nessa subescala, diferenciando-se bastante dos resultados encontrados no município de Erechim, também na Região Sul do Brasil, onde se verificou que em $94,2 \%$ dos lares investigados, a variedade de estimulação foi pontuada como muito boa. ${ }^{17}$

Na intenção de se buscar explicações para esse fenômeno, procurou-se analisar se crianças mais velhas ou adultos no ambiente doméstico poderia intervir na variedade de estimulação como pode ser verificado na Tabela 3.

A Tabela 3 mostra um nítido equilíbrio na amostra com a prevalência de uma a duas crianças no ambiente doméstico, o mesmo pôde ser observado no que diz respeito ao número de adultos, quando se constatou a existência de apenas dois. Esses dados sugerem que não havendo diferenças quanto ao número de crianças $(p=0,714)$ ou adultos $(p=0,696)$ no ambiente doméstico que explicasse o melhor desempenho do Grupo A no item variedade de estímulos ambientais, pode ser que a justificativa para os resultados supracitados apoie-se no fato de que nos lares onde se evidenciam maior renda, tanto o pai como a mãe trabalham, e, sendo assim, as crianças geralmente ficam aos cuidados de uma adolescente que na maioria das vezes está envolvida em outros afazeres domésticos, restringindo desta forma o tempo, a quantidade e a qualidade de estimulação.

Os resultados mais preocupantes deste estudo estão relacionados aos itens que tratam dos materiais que estimulam o de- 
senvolvimento da motricidade. Assim, independente do nível econômico, ambos os grupos obtiveram resultados catastróficos, pois $99 \%$ do grupo A e $100 \%$ do grupo B não atenderam ao critério de referência para os materiais que promovem o desenvolvimento da motricidade grossa; $99 \%$ e $96,2 \%$ apresentaram classificação fraca e muito fraca nos grupos A e B, respectivamente, no que diz respeito aos materiais que objetivam desenvolver a motricidade fina. Esses resultados corroboram com o estudo de Schobert ${ }^{17}$ que também evidenciou nas subescalas do AHEMD as piores classificações - 88,4\% e 100\% das residências não atendem aos critérios propostos pelo

Tabela 3: Distribuição de freqüência do número de crianças e adultos no ambiente doméstico.

\begin{tabular}{cccccccc}
\hline & GRUPO & $\mathbf{1}$ & $\mathbf{2}$ & $\mathbf{3}$ & $\mathbf{4}$ & $\mathbf{5}$ ou mais & $\mathbf{p}$ \\
\hline Número de & $\mathrm{A}$ & $(42)$ & $(44)$ & $(09)$ & $(03)$ & $(05)$ & \\
crianças & & $40,8 \%$ & $42,7 \%$ & $8,7 \%$ & $2,9 \%$ & $4,9 \%$ & \\
residentes & $\mathrm{B}$ & $(09)$ & $(14)$ & $(02)$ & $(01)$ & - & 0,714 \\
& & $34,6 \%$ & $53,8 \%$ & $7,7 \%$ & $3,8 \%$ & - & \\
& \multirow{2}{*}{ TOTAL } & $(51)$ & $(58)$ & $(11)$ & $(04)$ & $(05)$ & \\
& & $39,5 \%$ & $45,0 \%$ & $8,5 \%$ & $3,1 \%$ & $3,9 \%$ & \\
Número de & $\mathrm{A}$ & $(02)$ & $(51)$ & $(16)$ & $(19)$ & $(15)$ & \\
adultos & & $1,9 \%$ & $49,5 \%$ & $15,5 \%$ & $18,4 \%$ & $14,6 \%$ & \\
residentes & $\mathrm{B}$ & $(01)$ & $(12)$ & $(02)$ & $(05)$ & $(06)$ & 0,696 \\
& & $3,8 \%$ & $46,2 \%$ & $7,7 \%$ & $19,2 \%$ & $23,1 \%$ & \\
& \multirow{2}{*}{ TOTAL } & $(03)$ & $(63)$ & $(18)$ & $(24)$ & $(21)$ & \\
& & $2,3 \%$ & $48,8 \%$ & $14,0 \%$ & $18,6 \%$ & $16,3 \%$ & \\
\hline
\end{tabular}

* Diferenças significativas para $\mathrm{p}<0,05$ (teste Qui-quadrado)

AHEMD para materiais de motricidade fina e grossa, respectivamente.

Ao se constatar que o microssistema lar não atende às necessidades de ofertar oportunidades para promover o desenvolvimento motor das crianças, entende-se que a participação ativa delas em outros microssistemas em que isso aconteça é imprescindível. No entanto, como pode ser verificado na tabela 4 , independentemente do nível socioeconômico, em ambos os grupos existem crianças que nunca frequentaram o microssistema creche.

Os achados registrados neste trabalho alertam para o fato de que a baixa oportunidade de estímulos ambientais poderá comprometer o bom desenvolvimento motor das crianças, visto que na faixa etária deste estudo, encontram-se em estágios de desenvolvimento em que a relação entre o ser humano e o ambiente determinam o futuro comportamento motor da criança. ${ }^{2,18,19}$ Assim, estudos que procuram associar os estímulos ambientais e o desenvolvimento motor, por exemplo, testemunham a importância de materiais de jogos estimulantes como preditores do desenvolvimento. ${ }^{8,20}$

Em relação ao indicador AHEMD, total do somatório dos valores estandardizados que representa a quantidade e qualidade das 
affordance, obtiveram classificação baixa no ambiente doméstico 54,4\% do Grupo A e 50\% do Grupo B. Das residências pesquisadas, $53,5 \%$ tiveram uma pontuação baixa e 46,5\% média, não sendo encontrado em nenhuma re- sidência um nível alto de oportunidades para o desenvolvimento motor das crianças. O teste Qui-Quadrado apontou diferenças significativas a favor do grupo com renda mensal superior a $\mathrm{R} \$ 1.000$, nas variáveis espaço exterior

Tabela 4: Distribuição de freqüência do tempo de permanência das crianças em creches.

\begin{tabular}{lcccccc}
\hline & GRUPO & $\begin{array}{c}\text { Nunca } \\
\text { frequentou }\end{array}$ & $\begin{array}{c}\text { Menos de 6 } \\
\text { meses }\end{array}$ & $\begin{array}{c}6 \text { a 12 } \\
\text { meses }\end{array}$ & $\begin{array}{c}\text { Mais de 12 } \\
\text { meses }\end{array}$ & P \\
\hline Tempo em & A & $(59)$ & $(18)$ & $(18)$ & $(08)$ & \\
creche ou escola & & $57,3 \%$ & $17,5 \%$ & $17,5 \%$ & $7,8 \%$ & \\
& B & $(15)$ & $(03)$ & $(06)$ & $(02)$ & 0,849 \\
& & $57,7 \%$ & $11,5 \%$ & $23,1 \%$ & $7,7 \%$ & \\
& TOTAL & $(74) 57,4 \%$ & $(21) 16,3 \%$ & $(24) 8,6 \%$ & $(10) 7,8 \%$ & \\
\hline
\end{tabular}

* Diferenças significativas para $\mathrm{p}<0,05$ (teste Qui-quadrado)

$(p=0,013)$ e espaço interior $(p=0,022)$. No entanto, o teste U de Mann-Whitney não apontou diferenças significativas quando comparados os grupos em relação ao escore total ( $\mathrm{p}=$ 0,743) com médias 9,1 $\pm 1,7$ dp e 9,5 $\pm 2,2$ dp para os grupos A e B, respectivamente.

Os achados do presente estudo sugerem a necessidade de que seja realizada a avaliação do desenvolvimento motor das crianças para verificar as possíveis repercussões dos baixos níveis de estimulação ambiental constatadas nos microssistemas lar de Juazeiro do Norte, Ceará, Brasil, visto que estudos apontam para uma associação entre affordances e desenvolvimento motor. ${ }^{21}$ Assim, espera-se que tão logo seja confirmado o problema, desenvolvam-se estratégias e políticas públicas que revertam esse quadro e propicie uma melhor qualidade de vida para as crianças daquele município desde as mais tenras idades.

Aceita-se no meio científico a importância que o nível socioeconômico pode exercer sobre a quantidade e a qualidade dos estímulos ambientais direcionados a promover o desenvolvimento motor das crianças. Tal justificativa apoia-se no fato de que as famílias com uma maior renda teriam, por conseguinte, uma melhor capacidade de adquirir residências com um perfil arquitetônico que conduzisse a uma maior quantidade de affordances em função da estruturação dos espaços exterior e interior. $\mathrm{O}$ presente estudo reproduz outros resultados. Mostra que há uma baixa organização do espaço exterior como fator de promoção do desenvolvimento motor, sugerindo, assim, a necessidade de uma aproximação das áreas de arquitetura, engenharia civil e educação física, pois juntas poderão encontrar solução para esse problema.

Apesar de a literatura afirmar que o nível intelectual do agente mediador é determinante na variedade de estímulos ambientais presentes no microssistema lar, os achados deste estudo contradizem essas afirmativas, apontando, inclusive, um melhor desempenho das famílias com menor nível econômico e cultural nessa variável. Tal fato pode ser justificado em função de que nas famílias com menor renda geralmente apenas o pai trabalha, ficando os cuidados das crianças entregues às mães; nos lares de maior renda, tanto o pai quanto a mãe trabalham, ficando geral- 
mente as crianças entregues aos cuidados de uma babá, que na maioria das vezes é adolescente e ocupada com outros afazeres domésticos, restringindo-se assim a quantidade e a qualidade de variedade de estímulos ambientais.

O fato mais preocupante neste estudo diz respeito à disponibilidade de materiais que estimulem o desenvolvimento das habilidades motoras grossas e finas das crianças, visto que a literatura afirma que um dos fatores que mais influencia o desenvolvimento motor das mesmas é a disposição de brinquedos e materiais de jogos. Assim, ao se constatar que nem mesmo as famílias com melhor nível socioeconômico conseguiram uma classificação aceitável para essa variável, tudo leva a crer que existe uma possibilidade para que o profissional de

\section{REFERÊNCIAS}

1. Haywood KM, Getchell N. Desenvolvimento motor ao longo da vida. $3^{\mathrm{a}}$. ed. Porto Alegre: Artmed, 2004.

2. Gallahue DL, Ozmun JC.

Compreendendo o Desenvolvimento Motor: bebês, crianças, adolescentes e adultos. 3a. ed. São Paulo: Phorte, 2005.

3. Payne VG, Isaac LD. Desenvolvimento Motor Humano: uma abordagem vitalícia. $6^{\mathrm{a}}$. Ed., Rio de Janeiro: Guanabara Koogan, 2007.

4. Bronfenbrenner U, Morris PA. The Ecology of Developmental Process. In: I. Damon I, Lerner RM, organizadores. Handbook of child psychology: Theoretical models of human development. New York: John Wiley \& Sons; 1998. p. 992-1027.

5. Bronfenbrenner U, Morris PA. The Ecology of Developmental Process. In: Pedro JG, editor. Stress and Violence in Childhood and Youth. Lisboa: Faculdade de Medicina; 1999. p. 21-95.
Educação Física e de outras áreas relacionadas ao movimento atue nesse setor prestando consultoria na aquisição de materiais e orientando o uso dos mesmos no sentido de estimular o desenvolvimento motor das crianças.

Portanto, ao se constatar que, de maneira geral, as affordances presentes no microssistema lar se mostram ineficientes para promover o desenvolvimento motor das crianças, o estudo sugere que há uma necessidade de investimentos na criação de creches e espaços públicos, e contratação de profissionais capacitados que atendam a essas necessidades.

6. Krebs RJ. A Criança e o Esporte: Reflexões Sustentadas pela Teoria dos Sistemas Ecológicos. In: Krebs RJ, Beltrame TS, Copetti F, Pinto RF, organizadores. Os Processos Desenvolvimentais na Infância. Belém: GTR; 2003. p. 91-104.

7. Rodrigues L, Gabbard C. Avaliação das oportunidades de estimulação motora presentes na casa familiar: Projecto affordances in the home environment for motor development. In Barreiros J, Cordovil R, Carvalheira S, editores. Desenvolvimento Motor da Criança. Lisboa: Edições FMH; 2007. p. 51-60.

8. Gabbard C, Caçola P, Rodrigues L. (2008). A New Inventory for Assessing Affordances in the Home Environment for Motor Development (AHEMD-SR). Early Childhood Educ. J. 2008; 36:5-9.

9. Bronfenbrenner U. The Ecology of Human Development: Experiments by Nature and Design. Cambridge, MA: Harvard University Press, 1979. 
10. Andrade SA, Santos DN, Bastos AC, Pedromônico MRM, Almeida-Filho N, Barreto M. Ambiente familiar e desenvolvimento cognitivo infantil: uma abordagem epidemiológica. Rev. Saúde Pública 2005; 39(4):606-611.

11. Muller AB. Efeitos da Intervenção Motora em Diferentes Contextos no Desenvolvimento da Criança com Atraso Motor [dissertação]. Porto Alegre: Programa de Pós-Graduação em Ciência do Movimento Humano da UFRGS; 2008.

12. IPECE (Instituto de Pesquisa e Estratégia Econômica do Ceará). Perfil Básico Municipal de Juazeiro do Norte. [internet]. 2007 [acesso em 10 jan 2009]. Disponível em: http:// www.ipece.ce.gov.br/publicacoes/ perfil_basico/PBM_2007/ Juazeiro\%20do\%20Norte.pdf

13. Rodrigues L. Development and validation of the AHEMD-SR (Affordances in the Home Environment for Motor Development-Self Report) [tese]. College Station: Department of Health and Kinesiology da Texas A\&M University; 2005.

14. Zajons R, Muller AB, Valentini NC. A influência de fatores ambientais no desempenho motor e social de crianças da periferia de Porto Alegre. Rev Educ Fís. 2008; 19(2):159-171.
15. Fleck AC, Wagner A. A mulher como a principal provedora do sustento econômico familiar. Psicol. Estud. 2003; 8:31-38.

16. Leite ATB, Souza MR. As desigualdades de sexo no mercado de trabalho de Goiânia [artigo]. In: Anais do XVI Encontro Nacional de Estudos Populacionais. Caxambu: ABEP, 2008.

17. Schobert L. O Desenvolvimento Motor de Bebês em Creches: um olhar sobre diferentes contextos. [dissertação]. Porto Alegre: Programa de Pós-Graduação em Ciência do Movimento Humano da UFRGS; 2008.

18. Piaget J. The origin of intelligence in children. New York: University Press, 1952.

19. Vigotsky LS. A formação social da mente. 6a . ed. São Paulo: Martins Fontes, 1999.

20. Rodrigues L, Gabbard C. Influência das oportunidades de estimulação motora presentes na casa familiar nos níveis de desempenho motor de crianças até aos 41 meses de idade [resumo]. In Anais do $2^{\circ}$ Congresso Internacional de Aprendizagem na Educação de Infância. Maia, 2007.

21. Rodrigues LP, Saraiva L, Gabbard C. Development and structural validation of an inventory for assessing affordances in the home environment for motor development. Res Q Exerc Sport. 2005; 76:140-148.

Recebido em: 05/03/2009 Modificado em: 20/03/2009 Aceito em: 27/03/2009 